\title{
How can tricyclic sophoridinic derivatives be used as autophagy inhibitors for cancer treatments?
}

\author{
"The tricyclic sophoridinic derivatives constitute a novel class of \\ autophagy inhibitors, which induce autophagy-dependent apoptosis \\ in cancer cells and indicate the potential value of antitumor drug \\ candidates."
}

First draft submitted: 23 March 2017; Accepted for publication: 30 March 2017; Published online: 21 June 2017

Keywords: anticancer $\bullet$ autophagy $\bullet$ sophoridinic

The tricyclic sophoridinic analog, $N$ '3,4,5trimethoxylbenzyl-12N-p-chlorobenzyl sophoridinamine $(\mathbf{6 b})$, blocks autophagic flux in tumor cells [1], severely suppresses lysosomal acidification and thereby inhibits tumor cell survival. The $12 N$-p-chlorobenzyl sophoridinic methyl ester IMB-6G-induced lysosomal membrane permeabilization (LMP) caused lysosomal release of cathespin B (CTSB)/ cathespin D (CTSD) and eventually triggered mitochondrial apoptosis [2]. The tricyclic sophoridinic derivatives constitute a novel class of autophagy inhibitors, which induce autophagy-dependent apoptosis in cancer cells and indicate the potential value of antitumor drug candidates.

Fufang Kushen injection, a traditional Chinese medicine, was approved by the China FDA in 1995 for treatment of nonsmall-cell lung cancer, liver cancer and gastric cancer [3-5]. It has been widely used for the treatment of diverse cancer patients in combination with other anticancer drugs, such as vinorelbine, cisplatin and taxol. Sophoridine as a quinolizidine alkaloid, one of the key active ingredients in Fufang Kushen injection, was also approved by the China FDA in 2005 to treat cancer patients with malignant trophoblastic tumors. However, the moderate antitumor activity of sophoridine limited its use as a drug for clinical applications, therefore, a lot of attention has been drawn for further development of its analogs to discover more effective drug candidates.

Taking sophoridine as the lead compound, we have constructed a compound library for tricyclic sophoridinic derivatives, and summarized the structure-activity relationship for antiproliferative activity of this kind of compounds. We have successfully identified that the $12-N-p$-chlorobenzyl sophoridinic derivatives with a tricyclic core were more favorable than sophoridine with a four-ring scaffold [1,2,6-8]. Moreover, the tricyclic sophoridinic derivatives own multiple druglike properties such as structural flexibility, high solubility and good pharmacokinetic and safety profiles, and constitute a novel family of potent antiproliferative agents. The representative compounds $\mathbf{6 b}$ and IMB-6G demonstrated a potent antitumor activity against a variety of human carcinoma cell lines. Especially, both of them displayed a promising potency against both wild type and drug-resistant carcinoma cell lines.

Autophagy is a highly conserved process where cells get rid of unwanted cellular components including damaged organelles, lipid vesicles or protein aggregates through lysosomal degradation [9]. It is a dynamic process
Yan-Xiang Wang

Institute of Medicinal Biotechnology, Chinese Academy of Medical Science \& Peking Union Medical College, Beijing 100050, China

Zhan-Dong Liu

Department of Neurology, Beijing Friendship Hospital, Capital Medical University, Beijing 100050, China

Chong-Wen Bi

'Institute of Medicinal Biotechnology, Chinese Academy of Medical Science \& Peking Union Medical College, Beijing 100050, China Lu Liu

'Institute of Medicinal Biotechnology, Chinese Academy of Medical Science \& Peking Union Medical College,

Beijing 100050, China

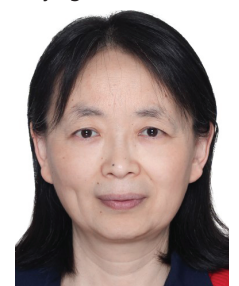

Dan-Qing Song

Author for correspondence:

Institute of Medicinal Biotechnology, Chinese Academy of Medical Science \& Peking Union Medical College, Beijing 100050, China

Tel.: +861063165268

Fax: +861063165268

songdanqingsdq@hotmail.com

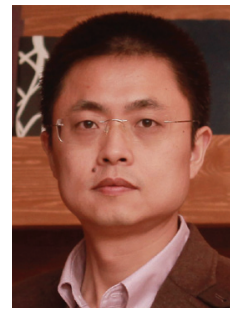

Hong-Bin Deng

Author for correspondence:

Institute of Medicinal Biotechnology, Chinese Academy of Medical Science \& Peking Union Medical College, Beijing 100050, China

Tel.: +861063169876

Fax: +86 1063017302

hdeng@imb.pumc.edu.cn

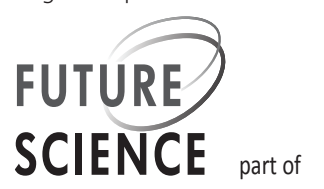


in which useless cellular components get sequestered in double-membranous vesicles called autophagosomes. Autophagosomes fuse with lysosomes to generate autolysosomes, in which the autophagic cargo is degraded by acidic hydrolases and released into the cytosol [10].

\section{"On the basis of the upregulation of autophagy in cancer cells treated with chemotherapeutic agents, autophagy inhibition has become a promising strategy for cancer treatment."}

Our previous studies demonstrated that tricyclic sophoridinic derivatives showed a potent antitumor activity via inhibiting the DNA topoisomerase I activity, inducing cell-cycle arrest at the G0/G1 phase, and cause apoptotic cell death in tumor cells, similar to that of parent sophoridine [4-6]. However, little is known about the ability of this kind of compounds to affect autophagy in tumor cells. Recently, we identified 6b and IMB-6G, as a novel autophagy inhibitor with chemical structure distinct from other known ones. Both of them inhibit the growth of A549, HepG2, MiaPaCa-2 and HupT-3 cancer cells and induce caspase-mediated apoptosis. Meanwhile, treatment with 6b and IMB-6G leads to substantial accumulation of both LC3-II and p62/SQSTM1 in human cancer cells, which indicated the blockade of autophagy by 6b and IMB-6G. LC3 turnover assay and mCherryEGFP-LC3 confocal microscopy assay also confirmed that the autophagic flux was indeed blocked by $\mathbf{6 b}$ and IMB-6G, these results thus support the conclusion that the inhibition of autophagy has a central role in tricyclic sophoridinic derivatives-mediated apoptotic cell death.

On the basis of the upregulation of autophagy in cancer cells treated with chemotherapeutic agents, autophagy inhibition has become a promising strategy for cancer treatment [11]. In autophagy, the lysosome is the only way to degrade cargos, so complete inhibition of the lysosome always causes serious impairment of autophagic flux. Low $\mathrm{pH}(\mathrm{pH}<4.5)$ is critical for the proteolytic activity in the lysosome [12]. Chloroquine (CQ) is a weak base that inhibits lysosomal acidification, which prevents the fusion of autophagosomes with lysosomes and subsequent autophagic degradation. Currently, CQ and its derivative hydroxychloroquine are widely used as late-stage autophagy inhibitors and have been evaluated in several Phase I and II clinical trials in combination with chemotherapy drugs in a variety of tumor types [13,14]. However, the safety concerns, including ocular toxicity and irreversible retinopathy limit their clinical use, and thereby it is highly desirable for new inhibitors of autophagy [15].
Sophoridinamine $\mathbf{6 b}$ is a basic nitrogen-containing compound, mainly resulting from the secondary amine $(\mathrm{NH})$ at the side chain, which makes the solution of it alkaline. It thus can be protonated and accumulates in lysosomes, resulting in the deacidification of their lumens. Similar to $C Q$, our finding demonstrate that $\mathbf{6 b}$ is a potent lysosomal-alkalizing agent that exhibits antitumor activity in various human tumor cell lines. Compound $\mathbf{6 b}$ actually blocks rather than activates autophagic flux in tumor cells, severely suppresses lysosomal acidification, thus preventing the activation of lysosomal enzymes and thereby their ability to degrade the cargo of the autolysosome, which in turn leads to a blockade of autophagic flux and suppress tumor cell survival [8].

In addition, sophoridinic ester IMB-6G promotes autophagosome accumulation from the early stage of treatment but blocks autophagic flux in the degradation stage, mainly through attenuation of lysosomal cathepsin activity in pancreatic cancer cells [16]. Compound IMB6G caused LMP in pancreatic cancer cells after treatment for $12 \mathrm{~h}$. Induction of LMP has been abundantly linked to cell death [17]. This suggests that LMP may have occurred after autophagic flux inhibition in IMB6G-treated pancreatic cancer cells. Furthermore, knocking down Atg5 alleviated IMB-6G-induced LMP and the abrogation of cathepsin activity by CA- $074 \mathrm{Me}$ and E-64 attenuated cell death in the presence of IMB-6G, implying that increased LMP is involved in the deathpromoting effects of IMB-6G. On the other hand, the increased levels of CTSB/CTSD in cytosolic extracts confirmed the IMB-6G-induced LMP in pancreatic cancer cells. Elevated levels of cytosolic CTSB/CTSD were detected after treatment with $5 \mu \mathrm{M}$ IMB-6G for $12 \mathrm{~h}$ in the cells. Inhibition of autophagosome formation with siRNA against Atg5 attenuated IMB-6G-induced lysosomal release of CTSB/CTSD. CTSB/CTSD is an important mediator of apoptosis after LMP [18]. Upon LMP, the released CTSD induces conformational change of Bax and its translocation to the mitochondria, subsequently promoting apoptosis [19]. Previously, we demonstrated that IMB-6G induced mitochondrial apoptosis through releasing cytochrome c into the cytosol and Bax translocation into mitochondrial [20]. Our data indicated that IMB-6G-induced LMP caused lysosomal release of CTSB/CTSD and eventually triggered mitochondrial apoptosis.

Altogether, our findings demonstrate that sophoridinamine $\mathbf{6 b}$ blocks autophagic flux in tumor cells, severely suppresses lysosomal acidification, and thereby inhibiting tumor cell survival, and sophoridinic ester IMB-6G-induced LMP caused lysosomal release of CTSB/CTSD and eventually triggered mitochondrial apoptosis. The tricyclic sophoridinic derivatives constitute a novel class of autophagy inhibitors, which induce autophagy-dependent 
apoptosis in cancer cells and indicate the potential value of antitumor drug candidates.

\section{Financial \& competing interests disclosure}

This work was supported by the National Natural Science Foundation of China (81321004 and 81473248). The authors have no other relevant affiliations or financial involvement with any organization or entity with a financial interest in or financial conflict with the subject matter or materials discussed in the manuscript apart from those disclosed.

No writing assistance was utilized in the production of this manuscript.

\section{References}

1 Bi CW, Zhang N, Yang P et al. Synthesis, biological evaluation, and autophagy mechanism of $12 \mathrm{~N}$-substituted sophoridinamines as novel anticancer agents. ACS Med. Chem. Lett. 8(2), 245-250 (2017).

2 Bi CW, Zhang CX, Li YH et al. Novel N-substituted sophoridinol derivatives as anticancer agents. Eur. J. Med. Chem. 81, 95-105 (2014).

$3 \mathrm{Xu}$ W, Lin $\mathrm{H}$, Zhang $\mathrm{Y}$ et al. Compound Kushen Injection suppresses human breast cancer stem-like cells by downregulating the canonical Wnt/beta-catenin pathway. J. Exp. Clin. Cancer Res. 30(1), 103 (2011).

4 Zhao Z, Fan H, Higgins $\mathrm{T}$ et al. Fufang Kushen injection inhibits sarcoma growth and tumor-induced hyperalgesia via TRPV1 signaling pathways. Cancer Lett. 355 (2), 232-241 (2011).

5 Wang M, Liu CX, Dong RR et al. Safety evaluation of chinese medicine injections with a cell imaging-based multiparametric assay revealed a critical involvement of mitochondrial function in hepatotoxicity. Evid. Based Complement. Alternat. Med. 2015, 379586 (2015).

6 Li X, Zhao WL, Jiang JD et al. Synthesis, structure-activity relationship and biological evaluation of anticancer activity for novel N-substituted sophoridinic acid derivatives. Bioorg. Med. Chem. Lett. 21(18), 5251-5254 (2011).

7 Bi CW, Zhang CX, Li YH et al. Synthesis and biological evaluation of sophoridinol derivatives as a novel family of potential anticancer agents. ACS Med. Chem. Lett. 5(11), 1225-1229 (2014).

8 Bi CW, Ye C, Li YH et al. Synthesis and biological evaluation of 12-N-p-chlorobenzyl sophoridinol derivatives as a novel family of anticancer agents. Acta Pharm. Sin. B. 6(3), 222-228 (2016).

9 Anding AL, Baehrecke EH. Autophagy in cell life and cell death. Curr. Top. Dev. Biol. 114, 67-91 (2015).
10

Galluzzi L, Pietrocola F, Levine B et al. Metabolic control of autophagy. Cell 159(6), 1263-1276 (2014).

11 Rubinsztein DC, Gestwicki JE, Murphy LO et al. Potential therapeutic applications of autophagy. Nat. Rev. Drug Discov. 6(4), 304-312 (2007).

12 Saftig P, Klumperman J. Lysosome biogenesis and lysosomal membrane proteins: trafficking meets function. Nat. Rev. Mol. Cell Bio. 10, 623-635 (2009).

13 Rosenfeld MR, Ye X, Supko JG et al. A Phase I/II trial of hydroxychloroquine in conjunction with radiation therapy and concurrent and adjuvant temozolomide in patients with newly diagnosed glioblastoma multiforme. Autophagy 10(8), 1359-1368 (2014).

14 Rangwala R, Chang YC, Hu J et al. Combined MTOR and autophagy inhibition: Phase I trial of hydroxychloroquine and temsirolimus in patients with advanced solid tumors and melanoma. Autophagy 10(8), 1391-1402 (2014).

15 Tehrani R, Ostrowski RA, Hariman R et al. Ocular toxicity of hydroxychloroquine. Semin. Ophthalmol. 23, 201-209 (2008).

16 Liu L, Zhang N, Dou Y et al. Lysosomal dysfunction and autophagy blockade contribute to IMB-6G-induced apoptosis in pancreatic cancer cells. Sci. Rep. 7, 41862 (2017).

17 Repnik U, Hafner Cesen M, Turk B. Lysosomal membrane permeabilization in cell death: concepts and challenges. Mitochondrion 19 Pt A, 49-57 (2014).

18 Mrschtik M, Ryan KM. Lysosomal proteins in cell death and autophagy. FEBS J. 282(10), 1858-1870 (2015).

19 Castino R, Peracchio C, Salini A et al. Chemotherapy drug response in ovarian cancer cells strictly depends on a cathepsin D-Bax activation loop. J. Cell. Mol. Med. 13(6), 1096-1109 (2009).

20 Zhang N, Bi CW, Liu L et al. IMB-6G, a novel N-substituted sophoridinic acid derivative, induces endoplasmic reticulum stress-mediated apoptosis via activation of IRE1 $\alpha$ and PERK signaling. Oncotarget 7(17), 23860-23873 (2016). 